\title{
Special Issue on International Nonthermal Food Processing Workshop: FIESTA 2012
}

\author{
Roman Buckow $^{1} \cdot$ Pablo Juliano ${ }^{1}$
}

Published online: 12 June 2015

(C) UKCrown: CSIRO; Crown Copyright as represented by: CSIRO 2015

The first part of this Food Engineering Reviews Special Issue highlights outstanding review articles presented at the International Nonthermal Food Processing WorkshopFIESTA 2012, which was held in Melbourne, Australia, on October 16-17, 2012. Selected speakers were invited to submit manuscripts based on their presentations, which were then peer-reviewed in accordance with the standards of this journal. The workshop is an annual event organized by the Nonthermal Processing Division of the Institute of Food Technologists (IFT) and the European Federation of Food Science and Technology (EFFoST) in a number of countries around the world. In 2012, the workshop was hosted by the CSIRO, Australia's National Research Organization, as part of the biennial Innovative Foods Conference Series, FIESTA, where the theme was "Innovative Processes for Sustainable, Safe and Healthy Foods."

The 2-day workshop program featured more than 20 internationally renowned speakers and 40 poster displays to further advance science and technology on fundamentals and applications of nonthermal and other advanced food processing technologies. To add value and enable the sustainable manufacture of healthier food products was the primary focus of this event. Other relevant topics featured in the workshop included consumers, markets and sustainability, which are all essential elements for successful adoption and commercialization of emerging technologies.

The workshop was a major success attracting over 170 delegates from 18 countries and 86 organizations representing industry, academia and government. The workshop was also attended by several virtual delegates which followed presentations online and on Twitter.

We are delighted to include five high-quality reviews on nonthermal food processing where the emphasis is on food engineering aspects. These articles cover atmospheric pressure plasma, ultra-high-pressure homogenization (UHPH), high-pressure processing (HPP), megasonic separation and ultrasound processing. In particular, this Special Issue reviews the latest research on plasma applications on solid and liquid foods, the effects and novel applications of the UHPH process, the application of HPP in fish muscles, design aspects of megasonic separation reactors and the latest research on the application of ultrasound to conventional dairy processes.

We gratefully acknowledge the support of all workshop sponsors and thank all authors and reviewers for making possible this valuable, high-quality contribution to the body of knowledge on nonthermal processing of food.

Roman Buckow

roman.buckow@csiro.au

Pablo Juliano

pablo.juliano@csiro.au

1 CSIRO Food and Nutrition Flagship, Melbourne, Australia 